\title{
AUTOMATION AND WIRELESS COMMUNICATION TECHNOLOGIES IN ROAD REHABILITATION
}

\author{
Pekka Kilpeläinen \\ VTT Technical Research Centre of Finland \\ P.O.BOX 1100 (Kaitoväylä 1), \\ FIN-90571 Oulu FINLAND \\ Pekka.Kilpelainen@vtt.fi
}

\author{
Rauno Heikkilä \\ University of Oulu, Research Unit of \\ Construction Technology, POBOX 4400 \\ (Kiilakiventie 1) \\ FIN-90014 Oulu, FINLAND \\ Rauno.Heikkila@oulu.fi
}

\author{
Tommi Parkkila \\ VTT Technical Research Centre of Finland P.O.BOX 1100 (Kaitoväylä 1), \\ FIN-90571 Oulu FINLAND \\ Tommi.Parkkila@vtt.fi
}

\begin{abstract}
The goal of the project is to survey the applicability of machine automation and wireless communication technologies in road repairing and surfacing. The focus is on the control of the worksite and road repairing and road surfacing process. This process contains design, construction, quality control and work site management. The objective is to study, how different machines, e.g. asphalt mixing plant, trucks, paver and vibrating roller, can be equipped with wireless data connections and automation systems and what are the possibilities and benefits of ICT and machine automation in this application field. During year 2006 visits to the asphalt pavement repairing sites have been arranged. Different asphalt repairing methods as well as practices in production and transportation of asphalt mass have been studied. Persons that are working in asphalt work have been interviewed. Asphalt transportation and it's synchronisation to the asphalt spreading and asphalt production is one detected area of development. A concept of an asphalt process management system has been developed. The concept consists of connecting the trucks, asphalt pavers and the asphalt plant to a database by using wireless data communication. Process variables, such as mass temperature and transportation time, are measured in real time and saved to the database. From the information collected to a database an up-to-date view that shows the functioning of the whole process can been generated. With the better control of asphalt transportation the defects in pavement quality can be reduced. The goal is to test this concept by developing a prototype system and by arranging small scale field tests during year 2007.
\end{abstract}

\section{KEYWORDS}

Road Construction, Pavement, Automation, Wireless Communication

\section{INTRODUCTION}

In this research the focus was on repairing pavement failures on old roads and pavement laying on new roads. Much research work has been done automating different machines in road building and road rehabilitation [1]. In this research the focus was how a group of machines and a process they constitute can be managed better by applying ICT, embedded sensors and wireless communication technologies.

Pavement laying process consists of production of the asphalt mass, transportation of the mass to the work site, asphalt laying and compacting. When repairing old pavement, the old pavement material is usually recycled. This can be done using in-situ methods, e.g. using asphalt remixer. Other method 
is to use asphalt cutter to remove old surface and transport the crushed pavement to the asphalt mixing plant for recycling.

During this project many professionals working in asphalt production and spreading were interviewed. The co-ordination and synchronization of the asphalt production in the asphalt mixing plant, asphalt transportation and asphalt spreading in the work site was seen challenging. Many variables, e.g. weather, traffic conditions, failures of the machines, the number of trucks available and transportation distance, have an affect to the process.

In Finland mobile asphalt mixing plants are widely in use. Distance to the gravel storage place and environmental restrictions have to be taken into account when selecting the location of the asphalt mixing plant. On mobile asphalt mixing plants there are usually no silos for asphalt mass. So every asphalt batch is made directly to the loading pallet of the truck. The placement of the mobile asphalt mixing plant has to be chosen so that the transportation distance to the worksite doesn't exceed $100 \mathrm{~km}$. This ensures that that the asphalt mass doesn't cool too much during transportation.

Usually the asphalt mixing plant produces asphalt mass for more than one paver. It is important for asphalt mixing plant operator to know how each paver is progressing in order to adapt the production and transportation of the asphalt mass to the need of mass on each paver. In order to accomplish good pavement quality, asphalt spreading should be done without unnecessary stops. The transportation should work so that the paver doesn't have to wait for mass. On the other hand it is important that the truck doesn't have to wait for unloading so long time that the mass cools too much.

Also part of logistics of the asphalt production and pavement laying is transportation of the materials (bitumen, filler) and heavy fuel oil. Usually in the mobile asphalt mixing plant there are no containers for large amount of bitumen and oil, so bitumen and oil are transported to the mixing plant usually for the need of one workday. For this reason it is important to track the progress of the pavement laying and estimate the need of bitumen and oil for the next workday.
The conclusion was that with the better synchronization of asphalt production, transportation chain and asphalt spreading the performance of the whole process can be improved and a better pavement quality achieved. More upto-date information about how the process work is needed. This information should be shown illustrative form. In this paper a concept how asphalt process can be instrumented is described. Currently the development of the prototype is underway the first small scale tests will be done during year 2007 .

\section{CONCEPT OF AN ASPHALT PAVEMENT PROCESS MONITORING}

\subsection{Background}

In order to achieve good pavement quality the asphalt production, transportation, spreading and compacting must be working seamlessly. The hypothesis was that the process is similar to a normal plant with the difference that some of the production machines are outside of the fences of the plant. Nevertheless these machines can be connected to the plant by applying wireless communication. So trucks and asphalt pavers can be equipped with embedded measurement devices that measure process variables and these machines are connected to the asphalt mixing plant. This enables automatic data collection. Applying web technologies the collected data can be stored and formed to usable form for different users. Users have access to the data by using their personal mobile devices or computers.

VTT has previously tested mobile technology in "Wireless construction site" - project [2], where a system for managing mass transportation tasks in road construction was developed and tested. Truck drivers used their mobile phones to access and store data about transportation tasks to the database. System was based on Java applications in phones and GPRS (General Packet Radio Service) as communication technology. In this project the experiences of mobile technology were promising. One of the main advances was that data collecting can be done using very cheap and portable device. No constant installations to the trucks were needed. This makes easy for subcontractors to take the system into the use. 
One of the disadvantages discovered is that the system is totally dependent of the users. This makes the system prone to human errors and it depends much on motivation and carefulness of the user, how reliable and up-to-date the collected data is. Another problem with mobile phones is that they don't provide interfaces for adding sensors and measuring process variables.

At the same time embedded system and wireless communication technologies have developed so that even more simple embedded devices can be connected to the internet e.g. using GSM/GPRS modules and GPS positioning can be added to the embedded system. This enables cost efficient instrumentation of the mobile machines.

As a goal of the development work was to make the data collection and measurements of the process variables as automatic as possible and minimize the interaction needed from the users. Embedded measurement modules are used for data collection. VTT have already developed a measurement module for measuring vibrations from the work machines [3]. This module was used as a basis for developing a prototype system.

Compared to the mobile phone technology a disadvantage is that permanent installations to the machines are needed. Mobile phone technology and embedded measurement are not mutually exclusive. Mobile phone applications can be used for data collecting tasks that need user interaction and distributing information to the workers.

\subsection{Users}

Workers in the worksite need information that is relevant to their work task. The goal is to provide needed information in a visual form. The users in this case are:

- work supervisor

- $\quad$ asphalt mixing plant operator

- $\quad$ asphalt paver operators

- $\quad$ truck drivers

Work supervisor and asphalt mixing plant operator need information concerning the whole process, e.g. operating status of all the pavers as well as locations of the asphalt trucks. This enables for example allocation of the trucks between different pavers as well as tracking the progress of the paving. Operator of the asphalt paver needs information related to his own paver. Important information for him/her is for example when asphalt trucks are arriving to the paver. For truck drivers important information is the route to the paver as well as data needed for billing, e.g. total amount of transported mass, driving distances, waiting time etc. Example of a process view is shown in figure 1.

\subsection{Data collection and visualisation}

\subsubsection{Performance of the transportation chain}

The performance of the transportation chain can be evaluated by measuring how much time is spend in each part of the cycle of asphalt truck:

- $\quad$ Loading time [min]

- Transportation time [min]

- Waiting time for unloading [min]

- Unloading time [min]

- $\quad$ Returning time [min]

- Waiting time for loading [min]

Other measured variables are:

- Transportation distance $[\mathrm{km}]$

- Amount of transported asphalt mass [t]

From these measurements the instantaneous performance $[\mathrm{t} / \mathrm{h}]$ can be calculated. This can be compared to the theoretical performance achievable in the current traffic conditions. This can be estimated assuming the waiting times to be zero and trucks are transporting maximum loads. The key values that represent the performance of the transportation chain can be updated in real time, figure 1.

\subsubsection{Tracking of truck locations}

Tracking of the asphalt track locations is used for estimating the time when a truck arrives to the paver or returns to the asphalt mixing plant. Asphalt paver operator can decrease the speed of the paver to avoid stopping if he/she has the information that the next truck is delayed. Also asphalt production at the asphalt mixing plant can adapted to the number of returning trucks. If there are no trucks coming for a while, the asphalt plant can be shut down in order to save fuel. In some cases it is useful to wait several trucks to return and then restart the plant. This way unnecessary restarts can be avoided. 
Traffic conditions affect to the transportation time. Using GPS and time measurements the arriving time of the truck can be estimated if the transportation times of the previous trucks on the same route are known. Truck locations and estimated arriving times can be illustrated to the operators, figure 1.

\subsubsection{Measurement of the temperature of the asphalt mass}

The mass temperature should not be lower than $150{ }^{\circ} \mathrm{C}$ in order to accomplish good pavement quality. So system for monitoring cooling of the mass during transportation was seen important. This can be achieved by installing IR sensors to the trucks and pavers.

\subsubsection{Monitoring of the progress of the asphalt paving}

In order to monitoring the progress of the asphalt paving the paved distance and speed of the paver need to be measured. If a spreading width is also measured, paved area can be measured. If this information is available in the asphalt mixing plant, production and transportation can be adapted to the need of asphalt mass on different pavers.

\subsubsection{Registration of the truck loads}

During the paving the operator of the paver calculates the needed amount of asphalt mass and sends this information to the asphalt plant. The operator of the paver keeps track of transported amount of mass. Asphalt production on the asphalt plant is highly automated and information about each batch of mass is stored to the system of the plant. When GPS positioning is used in trucks, also information related to the transportation (e.g. unloading location, time, transportation time, temperature, truck ID) can be collected and stored. This information is useful for example when tracking reasons for quality defects or reporting done work to the customer.

\subsection{Communications}

Both long range communication and short range communication technologies are utilized in the system. Long range communication is needed for data transfer between embedded measuring devices and a server and for providing access to the server for users. GSM/GPRS services provided by telecommunication operators are used, figure 2 .

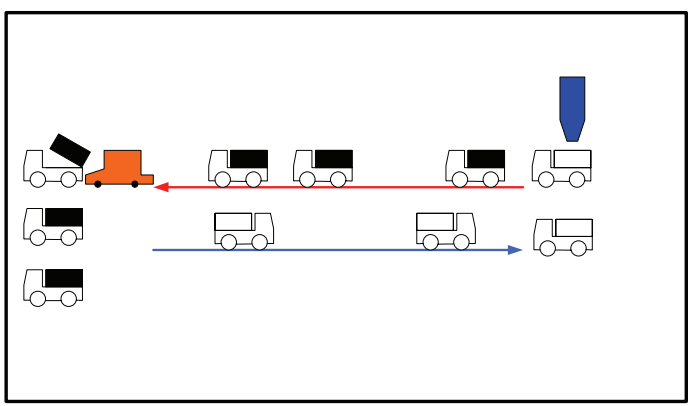

Figure 1 An Example of a Process View

Short range communication is needed for establishing a direct data transfer link between two machines. In this case a truck can connect directly to the asphalt mixing plant during loading and to the paver during unloading. This makes possible to transfer the information related to the asphalt load (load ID, amount of mass) from the asphalt mixing plant to the paver with the truck. Short range communication link can offer a backup system if GPRS service is unavailable, Figure 2.

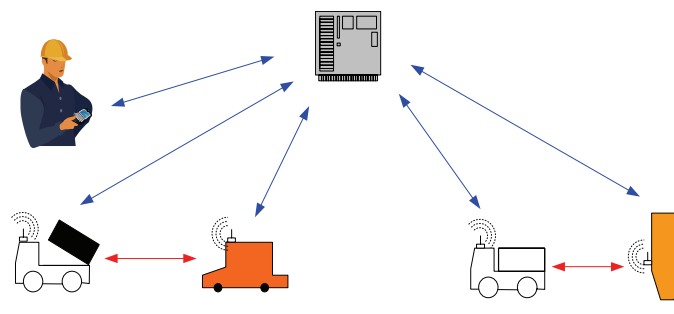

Figure 2 Short and Long Range Communication Technologies in Pavement Process Control

It is considered that for all special cases it not necessary to develop a mobile application. For example a truck driver can use a shortwave radio to inform the asphalt plant operator about delay or a technical problem. Asphalt plant operator then inputs this information to the system. After that the information is available to all who need it. 


\section{PROTOTYPE SYSTEM}

\subsection{Prototype System Overview}

Currently the development of a prototype system based on concept described above is on a way. Parts of the prototype are:

- Embedded measurement modules

- www server

- Web-based user interfaces for workers

Communication between measurement modules and server is done using a standard XMPP protocol [4]. XMPP protocol is very lightweight and this makes it useful when connecting embedded devices to the internet [5]. On the server there is a XMPP server to which the XMPP clients are connected. PHP Agent is used for accessing the mySQL database. PHP is also used for generating dynamical web pages. Most of the data processing is done on the server. GPRS is used for wireless data transfer between measurement modules and the server, figure 3 .

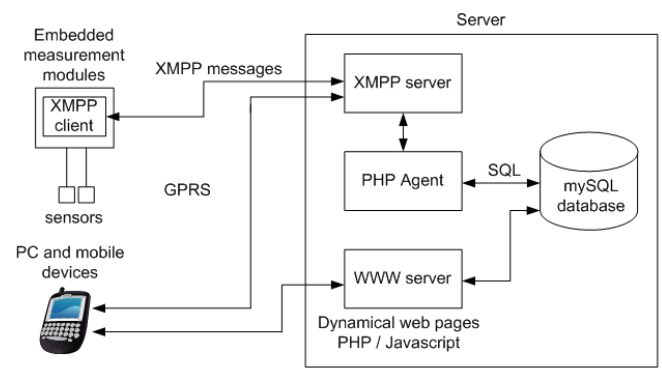

Figure 3 Prototype system overview

\subsection{Embedded Measurement Modules}

In figure 4 an embedded measurement module is shown. In the Module Atmel FPSLIC microcontroller is used. In this application an IR sensor (1) for measuring mass temperature, an inductive proximity sensor (2) for detecting unloading and a GPS positioning module (4) are connected to the module. Also some switches for the operator will be installed.

For communication both GPRS and nanoNET TRX radio modules (3) are installed inside the box. NanoNET TRX radio module from Nanotron Technologies $\mathrm{GmbH}$ is used for establishing a direct communication link between two machines.
NanoNET technology is suitable for harsh environments and VTT has used it in different industrial applications [6]. Technical challenge is to open the connection automatically without user intervention within a few seconds.

The task of the measurement module is to detect different events according to the programmed rules and if an event occurs send XMPP message to the server. Events can be detected by using:

- real time clock

- GPS position

- $\quad$ sensor data

- connection to the other machine

- request from server

For example if the arrival to the asphalt plant is detected according GPS position, a message to the server is send. Events are kept simple. Module ID, event ID, GPS position and time are always send and sensor values etc. are added to the message if needed. Server handles the event according to the event ID.

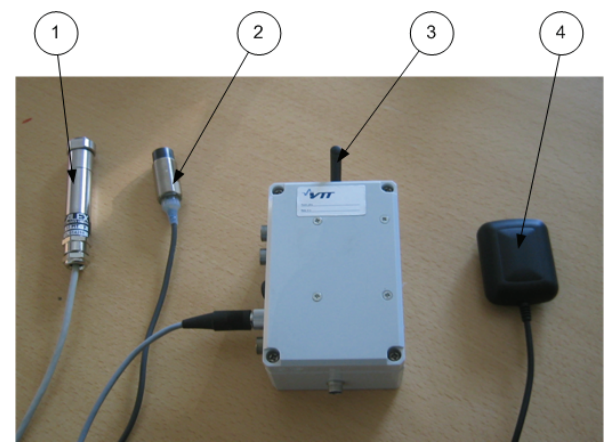

Figure 4 Embedded measurement module

\section{CONCLUSIONS}

In this paper a concept how ICT, embedded sensors and wireless communication technologies can be utilized in managing pavement laying process. Asphalt mixing plant, asphalt trucks and pavers are connected to each other using wireless technology. Embedded sensor modules are used for automatic data collection. Data is formed to a visual form so that overall performance of the process can be monitored. Decision making is still done by human operators, but the system provides them up-to-date information. 
There is always a risk that automatic data collection causes agony and is not accepted by the users. On the other hand if the system provides useful information in an easy form, it can reduce the workload and the stress of the operators. At present much data from the process is collected using handwritten forms. Processing handwritten forms can be laborious and slow. Automatic data collection can also reduce this work significantly.

Technical challenge is to achieve sufficient reliability. Also cheaper, smaller and easier to install measurement modules should be developed. Measurement modules should provided interfaces for various sensors and capability to use sensors that are already attached to the machine, e.g. through CAN bus interface. XMPP messaging is based on XML technology. Binary XML [7] is also a potential technology to enhance XMPP messaging, because it provides efficient data compression for XML data. The goal is to build a first prototype system and to test it during year 2007 and based on the experiences improve the system.

\section{REFERENCES}

[1] Kilpeläinen, P. Nevala, K.; Heikkilä, R,. (2004), Development of a control system for road construction automation applications, Proceedings of 21 st Int. Symposium on Automation and Robotics in Construction. Jeju, 21 - 25 Sep. 2004. pp. $65-70$
[2] Rannanjärvi, L., Känsälä, K (2003). Remote control and monitoring over Internet Wireless Construction Site, 7th IFAC Workshop on Intelligent Manufacturing Systems. Budapest, HU, 6 - 8 April 2003. Elsevier Science, pp. $205-210$

[3] Marjanen, Y. (2006). Long term measurements and analysis of day-to-day variability on whole body vibration exposure levels in work Environments. The Thirteenth International Congress on Sound and Vibration. Vienna, Austria, 2 - 6 July 2006.

[4] Saint-Andre, P., Extensible messaging and presence protocol (XMPP): Core, RFC 3920, $<$ : http://www.xmpp.org/specs/rfc3920.html, 7.6.2007.

[5] Parkkila, T. (2005), Application and platform management of an embedded system, Smart Systems 2005, Conference Proceedings. Seinäjoki, 3 - 4 May 2005.

[6] Keski-Säntti, J.,Parkkila, T., Leinonen, J., Leinonen, P. (2006), Wireless communication and mems sensors for cheaper condition monitoring and prognostics of charging crane. Proc. of the 19th International Congress of Condition Monitoring and Diagnostic Engineering Management, COMADEM 2006. Lulea University Press, pp. 747 - 755

[7] Efficient XML - Lightning fast Delivery of XML to More Devices in More Locations, URL: http://www.agiledelta.com/ product_efx.html, 7.6.2000. 